\title{
The effectiveness of electro-acupuncture combined with dyclonine hydrochloride in relieving the side effects of gastroscopy: a controlled trial
}

\author{
Jian-Ming Chen ${ }^{1,2}$, Dong-Dong $\mathrm{Li}^{3}$, Yi-Shan Chen ${ }^{1}$, Bo Lian ${ }^{1}$, Xiao-Peng Wang ${ }^{1}$, Yu-Hong Guo ${ }^{1}$, \\ Xiao-Long $\mathrm{Xu}^{1}$, Po Huang ${ }^{1}$, Teng-Fei Chen ${ }^{1}$, Yang Liu ${ }^{2}$, Qing-Quan Liu ${ }^{1}$ \\ ${ }^{1}$ Department of Intensive Medicine, Beijing Hospital of Traditional Chinese Medicine, Capital Medical University, Beijing, China; ${ }^{2}$ Department \\ of Gastroenterology, The 81th Group Army Hospital of the Chinese People's Liberation Army, Zhangiiakou, China; ${ }^{3}$ Department of Cardiology, \\ Tangdu Hospital, Air Force Medical University, Xi'an, China \\ Contributions: (I) Conception and design: JM Chen, QQ Liu; (II) Administrative support: JM Chen, QQ Liu, XL Xu; (III) Provision of study materials \\ or patients: Y Liu, P Huang, TF Chen, XP Wang; (IV) Collection and assembly of data: DD Li, B Lian; (V) Data analysis and interpretation: JM \\ Chen, YS Chen, DD Li, YH Guo, XL Xu, QQ Liu; (VI) Manuscript writing: All authors; (VII) Final approval of manuscript: All authors. \\ Correspondence to: Qing-Quan Liu. Department of Intensive Medicine, Beijing Hospital of Traditional Chinese Medicine, Capital Medical University, \\ No. 23 of the National gallery backstreet, Dongcheng District, Beijing 100010, China. Email: drliu_lqq1060@163.com.
}

Background: The present study aimed to explore the effectiveness of electro-acupuncture (EA) in combination with a local anesthetic used in Western medicine in preventing the side effects of gastroscopy.

Methods: A sample group of 150 patients were divided into three groups based on treatment methods: an EA group, a dyclonine hydrochloride mucilage group, and a combined treatment group. In the EA group, EA stimulation was given at the Hegu, Neiguan, and Zusanli acupoints; in the dyclonine hydrochloride mucilage group, patients took $10 \mathrm{~mL}$ of dyclonine hydrochloride mucilage orally; in the combined treatment group, prevention of side effects was attempted by administration of both acupuncture and oral local anesthetic. The incidences of nausea, emesis, salivation, cough, restlessness, and breath holding during gastroscopy were observed and recorded for the three groups. Mean arterial pressure, heart rate, and oxygen saturation were recorded before the examination, and changes in these measures were recorded as the gastroscope passed through the pylorus and after the examination. The visual analogue scale (VAS) values of nausea and emesis, the rate of successful first-pass intubation, and the time of gastroscopy were also recorded. Statistical analysis was performed using R-3.5.3 software.

Results: Incidences of side effects (e.g., nausea, emesis, salivation, restlessness, and breath holding) during the examination were lower in the combined treatment group than in the EA group and the dyclonine hydrochloride mucilage group $(\mathrm{P}<0.05$ and $\mathrm{P}<0.01$, respectively). Furthermore, the changes in heart rate and oxygen saturation when the gastroscope passed through the pylorus and after the examination were better in the combined treatment group than in the EA group and dyclonine hydrochloride mucilage group $(\mathrm{P}<0.01)$. The VAS values of nausea and emesis, the first-pass success rate, and examination duration were also better for the combined treatment group than for the other two groups $(\mathrm{P}<0.05$ and $\mathrm{P}<0.01)$.

Conclusions: EA combined with local anesthesia with dyclonine hydrochloride mucilage can alleviate side effects during gastroscopy, reduce patient pain, and improve the efficiency of the procedure.

Keywords: Side effects of gastroscopy; electro-acupuncture (EA); dyclonine hydrochloride mucilage; combined medication; preventive effect

Submitted Mar 27, 2020. Accepted for publication Dec 17, 2020.

doi: $10.21037 / \mathrm{apm}-20-831$

View this article at: http://dx.doi.org/10.21037/apm-20-831 


\section{Introduction}

Digestive endoscopy is a common method used in the diagnosis and treatment of digestive tract diseases (1) and for the early detection and treatment of organic lesions of the gastroesophageal mucosa. However, gastroscopy is often accompanied by physiological and psychological response and damage, for example, nausea $(2,3)$, emesis $(2,3)$, abdominal distension, abdominal pain, palpitation, tension, anxiety, dizziness, sore throat, elevated blood pressure, and even syncope; researchers refer to these effects collectively as "the side effects of gastroscopy" (4-6). The emergence of these side effects has brought difficulties to the operation and treatment of gastroscopy. A current intervention to relieve stress in clinic is psychological support; pharyngeal anesthesia (e.g., lidocaine) and sedation (e.g., diazepam, midazolam, or propofol) are also used. These can effectively reduce discomfort during a gastroscopy (7-10). However, the use of additional drugs increases the cost of surgery and may lead to nausea during induction of anesthesia (2). Furthermore, sedatives have associated risks of respiratory and cardiovascular inhibition, hypotension, and even coma $(11,12)$. The search for solutions to reduce side effects during examination and treatment is therefore a burning issue in the medical field. For a long time, traditional Chinese medicine has used acupuncture and moxibustion to relieve pain. In recent years, there have been many reports on the use of acupuncture, ear acupuncture, and electroacupuncture (EA) in anesthesia before gastroscopy (13-16), and good curative effects have been achieved.

The present study aimed to explore the effectiveness of EA in combination with a local anesthetic used in Western medicine in preventing the side effects of gastroscopy. We present the following article in accordance with the STRICTA reporting checklist (available at http://dx.doi. org/10.21037/apm-20-831).

\section{Methods}

\section{Study design and patients}

This prospective cohort study was carried out at the Outpatient Department of Gastroenterology, The 81 th Group Army Hospital of the Chinese People's Liberation Army. The study was conducted in accordance with the Declaration of Helsinki (as revised in 2013). The study was approved by the ethics committee of the 81th Group Army Hospital of the Chinese People's Liberation Army (No.: 2016JL03-013-01) and informed consent was taken from all the patients.

\section{Inclusion and exclusion criteria}

(I) Inclusion criteria: (i) patients between the ages of 20 and 50, inclusive; (ii) patients with indications for electronic gastroscopy; and (iii) patients who volunteered to participate in the present study and provided informed consent.

(II) Exclusion criteria: (i) patients whose ages fell beyond the scope of the study (i.e., younger than 20 or older than 50); (ii) patients with clear contraindications to gastroscopy; (iii) patients with neuropsychiatric disorders, severe anxiety and depression, or incapable of civil conduct; (iv) patients with serious or underlying diseases, such as diseases of the heart, brain, kidney, hematopoietic system, and respiratory system, which may induce abnormally related indicators during the gastroscopy process; (v) patients who could not accept acupuncture and EA, and are not allergic to acupuncture and moxibustion; and (vi) women who were pregnant or lactating.

\section{Intervention methods}

Patients were assigned to one of three groups-EA, dyclonine hydrochloride mucilage (D), or combined treatment $(\mathrm{EA}+\mathrm{D})$-based on their already existent treatment.

Before the gastroscopy, all patients were required to undergo fasting and water deprivation for more than eight hours [Olympus Medical Company, National Food and Drug Supervision Instrument (Import) No. 32707, 2011]. Before the examination, all patients were given sufficient psychological reassurance to enable them to relax in body and mind and to avoid anxiety.

(I) EA group: at 20 minutes before the examination, patients in the EA group were asked to lie down, and routine local skin disinfection was performed at the patient's bilateral Hegu (LI4), Neiguan (PC6), and Zusanli (ST36) acupoints. Acupuncture needles (Huatuo Acupuncture and Moxibustion Needles, Suzhou Medical Supplies Factory Co., Ltd.; specifications: $\varphi 0.30 \times 25 \mathrm{~mm}$ and $0.30 \times 40 \mathrm{~mm}$ ) were used for direct percutaneous needling. After insertion, needles were stopped when a patient experienced soreness; it was important that the stimulation intensity could be tolerated by the patient. The stocks of the acupuncture needles at the bilateral Hegu and Zusanli acupoints were 


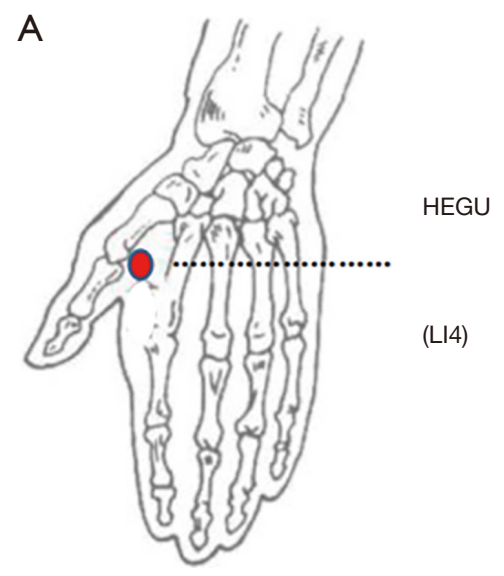

B
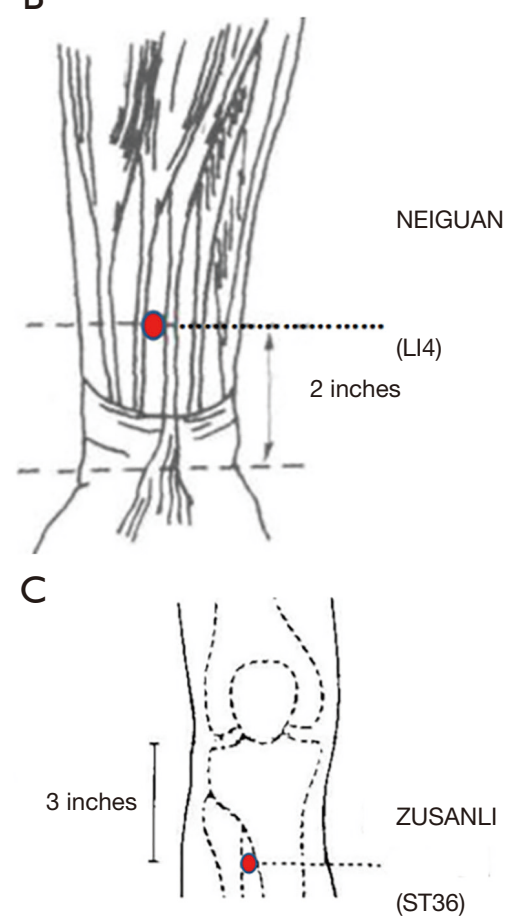

Figure 1 Anatomical location of acupoints: (A) Hegu; (B) Neiguan; (C) Zusanli.

connected with a Han stimulator [Nanjing Jisheng Medical Technology Co., Ltd., Jiangsu Food and Drug Supervision Instrument (Approval) No. 2260357, 2007]. Wires were connected to the ipsilateral acupoints, and a dilatational electrical wave (frequency: 2/15 Hz) was induced and maintained within a tolerable range for patients (the current was within 2-3 mA). The needles were retained in place until the end of gastroscopy; then, the electrical stimulation was stopped, and the needles were removed. The acupuncture was carried out by a practitioner with at least five years' experience.

Standard positioning (17):

Hegu (LI4): This acupoint is on the back of the hand at the midpoint of the radial side of the second metacarpal bone, between the first and second metacarpal bones (Figure 1A).

Neiguan (PC6): This acupoint is on the palm-side of the forearm between the long palmar muscle tendon and radial flexor muscle tendon of the wrist, two inches above the transverse crease of the wrist, at the connecting line of Quze and Daling (Figure 1B).

Zusanli (ST36): This acupoint is on the anterolateral calf at one finger width from the anterior margin of the tibia, three inches below acupoint Dubi (Figure 1C).

(II) $\mathrm{D}$ group: Before the gastroscopy, patients in the $\mathrm{D}$ group received $10 \mathrm{~mL}$ of oral dyclonine hydrochloride mucilage (Yangzijiang Pharmaceutical Group Co., Ltd., 10 mL/bottle; National drug approval: No. H20041523), swallowing it over the course of 15 minutes.

(III) $\mathrm{EA}+\mathrm{D}$ group: Patients in the $\mathrm{EA}+\mathrm{D}$ group were treated according to the two treatment methods in the EA group and the $\mathrm{D}$ group.

\section{Observation indexes}

(I) The incidences of nausea, emesis, salivation, cough, restlessness, and breath holding in the three groups during gastroscopy were observed and recorded.

(II) The rate of successful first-pass intubation was recorded.

(III)The time of gastroscopy was recorded; this was measured using a stopwatch (unit: minutes).

(IV)The mean arterial pressure (MAP), blood oxygen saturation $\left(\mathrm{SpO}_{2}\right)$, and heart rate $(\mathrm{HR})$ were recorded before the examination, as the gastroscope passed through the pylorus, and after the examination.

(V) The visual analogue scale (VAS) scores for nausea and emesis were recorded after the gastroscopy (18). A 10cm ruler was used as a scale plate: "0" was considered as indicating no nausea or emesis, while " 10 " indicated extremely serious nausea and emesis. This assessment was made by the patients immediately after the end of the gastroscopy; patients gave scores according to their individual feelings: the higher the score, the more serious their nausea and emesis. All VAS scores were evaluated by the same researcher, who had ten years of 
experience. This researcher was blinded to the group assignments.

\section{Recording and treatment of adverse reactions}

(I) Recording and observation of adverse reactions: clinical manifestations of adverse reactions, such as expression, complexion, nausea, emesis, salivation, cough, restlessness, and breath holding, were observed. Body temperature, rhythm, HR, blood pressure, respiratory rate, and $\mathrm{SpO}_{2}$ were recorded during the operation.

(II) Treatment of adverse reactions: if unforeseen events (e.g., patient fainting during acupuncture treatment, needle sticking, bending, or breaking, or hematoma) occurred during the acupuncture treatment, the patient either received specific treatment or was withdrawn from the study, according to the individual patient's symptoms and condition.

\section{Statistical analysis}

Data were statistically analyzed using R-3.5.3 software. The baseline data of the EA and D groups and of the EA+D group were evaluated using a Chi-squared test. A Chi-squared test was also used to analyze the side effects observed in patients during the examination. Intragroup paired samples comparison and intergroup independent samples comparison, before and after treatment, were conducted using $t$-tests. A nonparametric rank sum test was used to evaluate the overall curative effect between each pair of groups after treatment. Data were expressed as mean \pm standard deviation $(\bar{x} \pm \mathrm{SD})$.

\section{Results}

\section{General characteristics}

A total of 150 patients who were examined by gastroscopy in the Outpatient Department of Beijing Hospital of Traditional Chinese Medicine from June 2016 to December 2016 were enrolled in the present study. These patients were divided into three groups based on treatment methods: an EA group ( $\mathrm{n}=50)$, a dyclonine hydrochloride mucilage (D) group $(\mathrm{n}=50)$, and a combined treatment $(\mathrm{EA}+\mathrm{D})$ group $(n=50)$. The differences in gender, age, and weight, and past history of hypertension, diabetes, coronary heart disease, arrhythmia, gastrointestinal perforation, obstruction, acute and chronic liver diseases, acute and chronic biliary diseases, and acute and chronic pancreatic diseases were not statistically significant (inter-group comparison using a Chisquared test, $\mathrm{P}>0.05$, baseline alignment; see Table 1). There was no participant dropout during the study.

\section{Side effects in patients during gastroscopy}

The analysis reveals that incidences of most side effects after the gastroscopy, including nausea, emesis, salivation, restlessness, and breath holding, were lower in the EA+D group than in the EA and D groups, and the differences were statistically significant $(\mathrm{P}<0.05$ and $\mathrm{P}<0.01$, respectively). Although the frequency of cough was also lower in the EA+D group than in both the EA and D groups, the difference yielded in the intergroup comparison was not statistically significant $(\mathrm{P}>0.05)$. Comparing the two single-treatment groups, the incidence of side effects was lower in the EA group than in the D group; however, the difference between these two groups was not statistically significant $(\mathrm{P}>0.05)$. This indicates that, to some extent that, EA was better than dyclonine hydrochloride mucilage in preventing side effects in patients. Therefore, in the future, EA may be an alternative to drugs to alleviate the side effects of gastroscopy (Figure $2 A, B, C, D, E, F$ ).

\section{Comparison of MAP, $\mathrm{HR}$ and $\mathrm{SpO}_{2}$}

MAP, $\mathrm{HR}$ and $\mathrm{SpO}_{2}$ before the examination

Before the examination, the differences in $\mathrm{MAP}, \mathrm{SpO}_{2}$, and HR between the three groups were not statistically significant $(\mathrm{P}>0.05$; Figure 3$)$.

\section{MAP, $\mathrm{HR}$ and $\mathrm{SpO}_{2}$ during the examination}

As the gastroscope passed through the pylorus (i.e., during examination), MAP increased in the $\mathrm{EA}, \mathrm{D}$, and $\mathrm{EA}+\mathrm{D}$ groups. Although MAP was lower in the EA+D group than in the EA and D groups, the difference was not statistically significant $(\mathrm{P}>0.05)$. This result suggests that MAP was similarly elevated in all patients during the gastroscopy, and that none of the three treatment measures (EA, dyclonine hydrochloride mucilage, and a combination of the two) could effectively control its increase (Figure 4A).

$\mathrm{SpO}_{2}$ was increased in the EA, D, and $\mathrm{EA}+\mathrm{D}$ groups as the gastroscope passed through the pylorus (i.e., during examination). While $\mathrm{SpO}_{2}$ decreased to a certain extent in the $\mathrm{EA}+\mathrm{D}$ group after the gastroscope had passed through the pylorus, the level in this group remained significantly higher than the levels in the EA and $\mathrm{D}$ groups $(\mathrm{P}<0.01)$. 
Table 1 The general data of the three groups

\begin{tabular}{|c|c|c|c|c|}
\hline Items & \multicolumn{3}{|c|}{ Groups } & $\mathrm{P}$ \\
\hline$n$ & 50 & 50 & 50 & $>0.05$ \\
\hline \multicolumn{5}{|l|}{ Gender } \\
\hline Male & 26 & 24 & 28 & $>0.05$ \\
\hline Age & $30.24 \pm 7.01$ & $29.40 \pm 7.02$ & $29.52 \pm 7.01$ & $>0.05$ \\
\hline Weight & $66.86 \pm 6.02$ & $66.74 \pm 5.61$ & $66.11 \pm 5.92$ & $>0.05$ \\
\hline \multicolumn{5}{|l|}{ Past medical history } \\
\hline Hypertension & 4 & 4 & 2 & $>0.05$ \\
\hline Arrhythmia & 5 & 5 & 4 & $>0.05$ \\
\hline Gastrointestinal perforation & 2 & 0 & 1 & $>0.05$ \\
\hline Gastrointestinal obstruction & 0 & 0 & 0 & $>0.05$ \\
\hline Acute and chronic liver diseases & 8 & 10 & 7 & $>0.05$ \\
\hline Acute and chronic biliary diseases & 6 & 7 & 8 & $>0.05$ \\
\hline Acute and chronic pancreatic diseases & 2 & 3 & 2 & $>0.05$ \\
\hline
\end{tabular}

This suggests that, by preventing or retarding $\mathrm{SpO}_{2}$ depletion, the combined use of EA and orally administered dyclonine hydrochloride mucilage can better prevent the above-mentioned side effects in patients during examination than the use of EA or dyclonine hydrochloride mucilage alone (Figure 4B).

HR increased significantly in the EA, D, and EA+D groups as the gastroscope passed through the pylorus (i.e., during the examination). However, the changed HR was significantly lower in the EA+D group than in the EA and D groups $(\mathrm{P}<0.01)$. This suggests that the combined use of $\mathrm{EA}$ and orally administered dyclonine hydrochloride mucilage, by preventing or retarding the acceleration of HR in patients, can better prevent side effects during examination than the use of either treatment alone (Figure 4C).

\section{MAP, HR and $\mathrm{SpO}_{2}$ at the end of the examination}

At the end of the examination (i.e., gastroscope extraction), MAP increased in the EA, D, and EA+D groups. Although MAP was lower in the EA+D group than in the EA and $\mathrm{D}$ groups, the difference was not statistically significant $(\mathrm{P}>0.05)$. This suggests that MAP increased similarly in all patients upon gastroscope extraction after the end of the gastroscopy, and that none of the three treatment measures (EA, dyclonine hydrochloride mucilage, and a combination of the two) was able to effectively control this increase (Figure 5A).

$\mathrm{SpO}_{2}$ increased in the EA, D, and EA+D groups at the end of the examination (i.e., gastroscope extraction). Although $\mathrm{SpO}_{2}$ decreased to a certain extent in the EA+D group at gastroscope extraction, the level in this group remained significantly higher than the levels in the EA and $\mathrm{D}$ groups $(\mathrm{P}<0.01)$. This suggests that, by preventing or retarding the decrease in $\mathrm{SpO}_{2}$, EA combined with orally administered dyclonine hydrochloride mucilage may better prevent the above-mentioned side effects in patients during an examination than either treatment alone (Figure 5B).

HR significantly increased in all three groups at the end of the examination (i.e., gastroscope extraction); however, HR was significantly lower in the $\mathrm{EA}+\mathrm{D}$ group at gastroscope extraction than in the EA and $\mathrm{D}$ groups $(\mathrm{P}<0.01)$. This suggests that EA combined with orally administered dyclonine hydrochloride mucilage, by preventing or retarding the acceleration of $\mathrm{HR}$ in patients, may better 

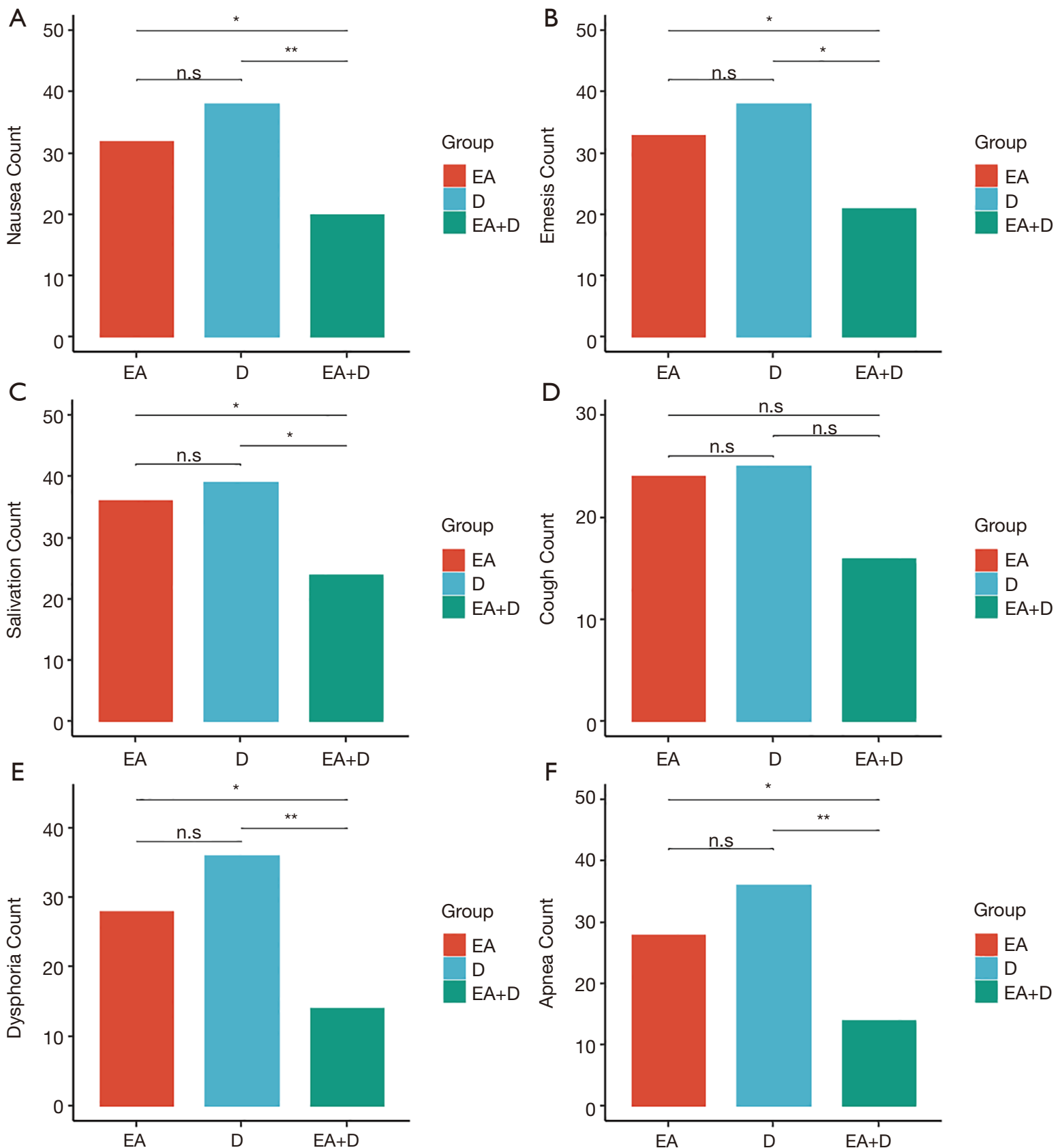
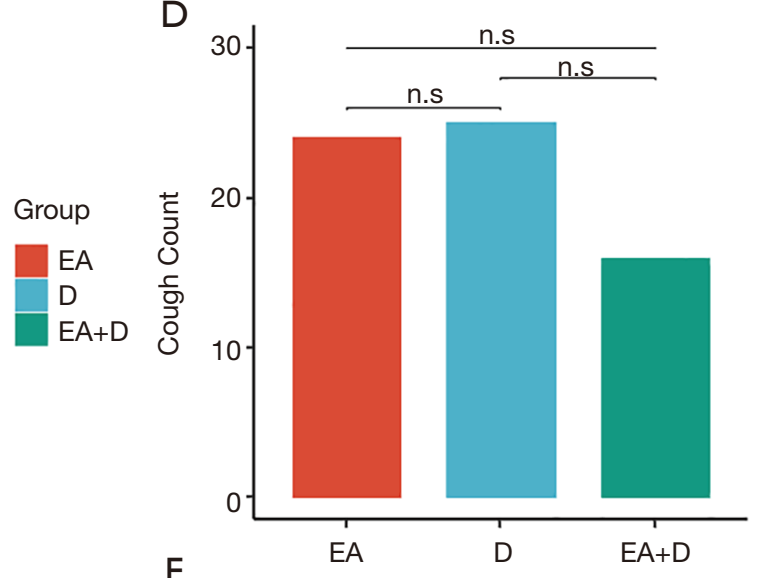

F
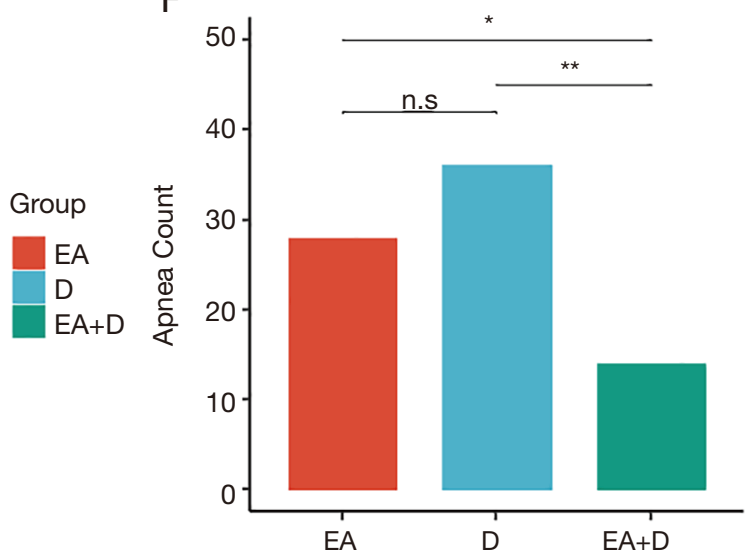
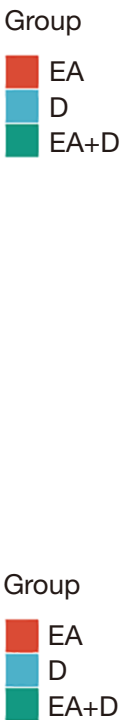

Figure 2 Comparison of incidences of side effects of gastroscopy among the three groups [n (number of patients)]: (A) Nausea, $\mathrm{PD}: \mathrm{EA}=0.53200$, PD: $\mathrm{EA}+\mathrm{D}=0.00702$, PEA:EA+D=0.04150; (B)emesis, PD:EA=0.6810, PD:EA+D=0.0133, PEA:EA+D=0.0410; (C) salivation, $\mathrm{PD}: \mathrm{EA}=1.000$, $\mathrm{PD}: \mathrm{EA}+\mathrm{D}=0.037$,PEA: $\mathrm{EA}+\mathrm{D}=0.037$; (D) cough, $\mathrm{PD}: \mathrm{EA}=1.000, \mathrm{PD}: \mathrm{EA}+\mathrm{D}=0.256, \mathrm{PEA}: \mathrm{EA}+\mathrm{D}=0.256$; (E) restlessness, PD:EA=0.297000, PD:EA+D=0.000348, PEA:EA+D=0.012700; (F) breath holding, PD:EA=0.297000, PD:EA+D= 0.000348,PEA:EA+D= 0.012700. These six values were expressed as n (patients). Among these, for the analysis of "nausea", "emesis", "salivation", "restlessness" and "breath holding": comparison of the EA+D group with the EA and D groups: ${ }^{*} \mathrm{P}<0.05$, ${ }^{* *} \mathrm{P}<0.01$; comparison of the EA and D groups: n.s. P $>0.05$; analysis of "cough": comparison of the EA+D group with the EA and D groups: n.s. P $>0.05$. 

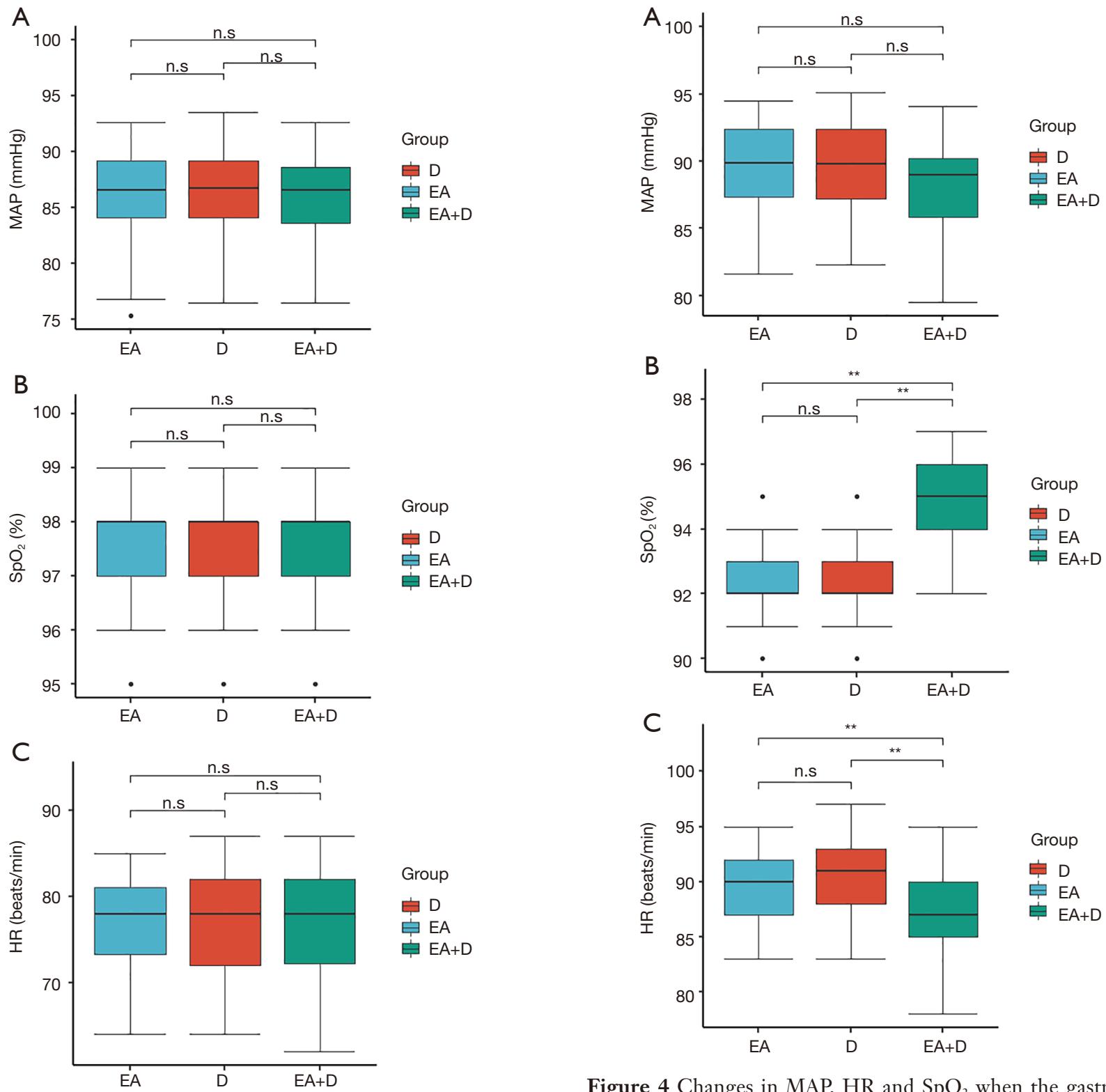

Figure 3 Changes in MAP, $\mathrm{HR}$ and $\mathrm{SpO}_{2}$ before the gastroscopy: (A) MAP (mmHg), $\mathrm{P}=0.9011$; (B) $\mathrm{SpO}_{2}$ (\%), $\mathrm{P}=0.6871$; (C) HR (sub/min), $\mathrm{P}=0.939$. All data were obtained using an ECG monitor, and all data results were expressed as mean \pm standard deviation $(\bar{x} \pm \mathrm{SD})$. Comparison among the three groups: n.s. $\mathrm{P}>0.05$.

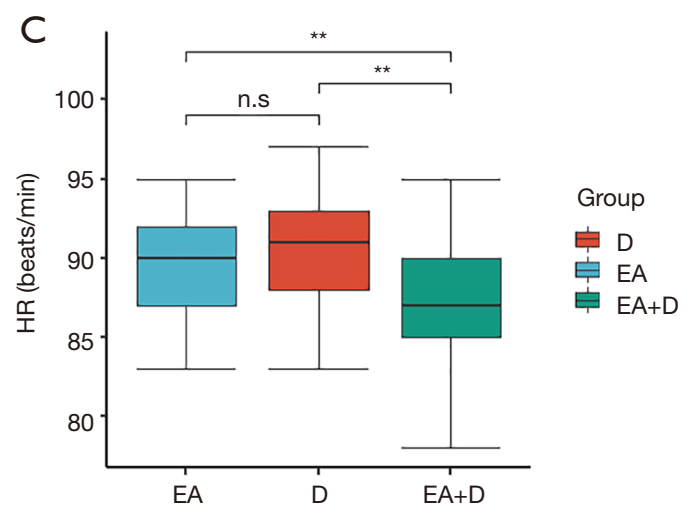

Figure 4 Changes in MAP, $\mathrm{HR}$ and $\mathrm{SpO}_{2}$ when the gastroscope passed through the pylorus (i.e., during gastroscopy): (A) MAP $(\mathrm{mmHg}), \mathrm{P}=0.05571$; (B) $\mathrm{SpO}_{2}(\%), \mathrm{PEA}-\mathrm{D}=0.54, \mathrm{PEA}+\mathrm{D}-\mathrm{D}$ $=1.4 \mathrm{e}-13, \mathrm{PEA}+\mathrm{D}-\mathrm{EA}=4.9 \mathrm{e}-14 ; \mathrm{P}=0.6871 ;(\mathrm{C}) \mathrm{HR}$ (sub/ min), PEA-D $=0.2958392$, PEA D-D $=0.0000007$, PEA D-EA $=0.0004984$. All data were obtained using an ECG monitor, and all data results were expressed as mean \pm standard deviation $\left(x_{ \pm} \mathrm{SD}\right)$. Comparison of MAP ( $\mathrm{mmHg}$ ) among the three groups: n.s. $\mathrm{P}>0.05$. Analysis of $\mathrm{HR}$ (sub/min) and $\mathrm{SpO}_{2}(\%)$ : comparison of the $\mathrm{EA}+\mathrm{D}$ group with the EA and $\mathrm{D}$ groups: ${ }^{* *} \mathrm{P}<0.01$; comparison of the EA and D groups: n.s. $\mathrm{P}>0.05$. 


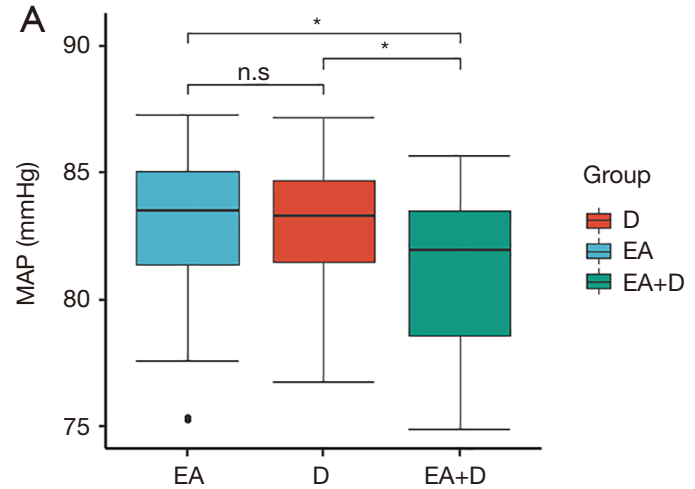

B

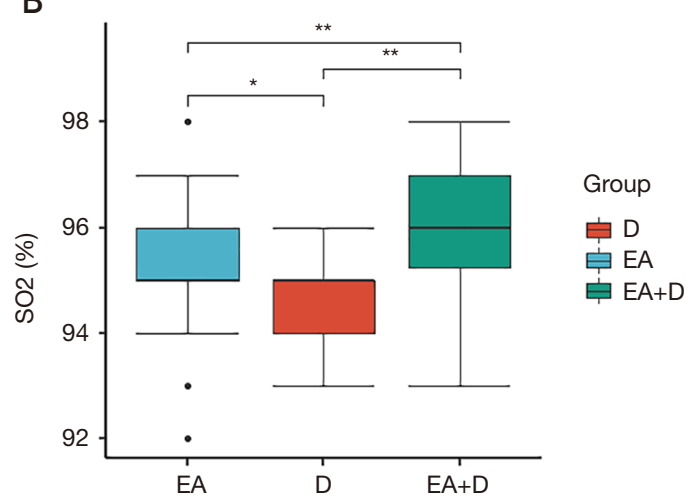

C

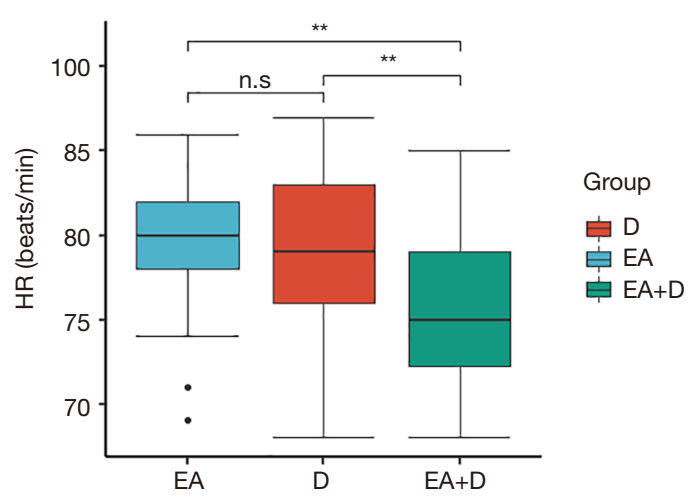

Figure 5 Changes in MAP, $\mathrm{HR}$ and $\mathrm{SpO}_{2}$ at the end of gastroscopy (i.e., gastroscope extraction): (A) MAP (mmHg), PEA-D=0.784, $\mathrm{PEA}+\mathrm{D}-\mathrm{D}=0.012, \mathrm{PEA}+\mathrm{D}-\mathrm{EA}=0.012$; (B) $\mathrm{SpO}_{2}$ (\%), PEA-D=0.01, $\mathrm{PEA}+\mathrm{D}-\mathrm{D}=3.2 \mathrm{e}-11, \mathrm{PEA}+\mathrm{D}-\mathrm{EA}=3.5 \mathrm{e}-06$; (C) $\mathrm{HR}$ (sub/min), PEA-D=0.56691, PEA+D-D=0.00015, PEA+D-EA=2.1e-06. All data were obtained using an ECG monitor, and all data results were expressed as mean \pm standard deviation $\left(\bar{x}_{ \pm} \mathrm{SD}\right)$. Comparison of MAP (mmHg) among the three groups: comparison of the $\mathrm{EA}+\mathrm{D}$ group with the EA and D groups: n.s. $\mathrm{P}>0.05$. Analysis of $\mathrm{HR}$ (sub/min) and $\mathrm{SpO}_{2}(\%)$ : comparison of the $\mathrm{EA}+\mathrm{D}$ group with the EA and D groups: ${ }^{*} \mathrm{P}<0.01$; comparison of the $\mathrm{EA}+\mathrm{D}$ group with the EA and D groups: n.s. $\mathrm{P}>0.05$. prevent side effects during examination (Figure 5C).

\section{Comparison of VAS scores of nausea and emesis among the three groups}

VAS scores of nausea and emesis after the examination The results of comparison between the three groups reveal that the VAS scores were lower in the EA+D group than in the EA and $\mathrm{D}$ groups $(\mathrm{P}<0.01)$, but that there was no statistical difference between the $\mathrm{D}$ and $\mathrm{EA}$ groups $(\mathrm{P}>0.05$; Figure 6).

\section{Comparison of the successful number of one-stop intubations among the three groups}

For 45 patients in the EA+D group, the gastroscopy was completed at the first pass: a success rate superior to those of the EA group (31 patients) and the D group (36 patients) $(\mathrm{P}<0.05)$. These results suggest that the combined treatment intervention can improve patients' tolerance of and compliance with gastroscopy (Figure 7).

\section{Comparison of duration of gastroscopy among the three groups}

Gastroscopy duration was shorter in the $\mathrm{EA}+\mathrm{D}$ group than in the $\mathrm{EA}$ and $\mathrm{D}$ groups $(\mathrm{P}<0.01)$. This suggests that the combined treatment intervention of $\mathrm{EA}$ and dyclonine hydrochloride mucilage may not only improve patients' tolerance to gastroscopy and reduce the side effects, but also help in shortening the duration of the procedure (Figure 8).

\section{Adverse events}

No adverse events and no events necessitating termination of the study (syncope, gastrointestinal bleeding, cardiocerebrovascular complications, perforation, or death) were observed in any of the three groups, and no patients withdrew from the study. No adverse events were related to the intervention.

\section{Discussion}

The first side effects of gastroscopy have their source in somatic stress, in which the physical stimulation of the inner wall of the throat and of the upper gastrointestinal tract causes pharyngeal and tension reflex, resulting in nausea and emesis. This also leads to psychological stress 


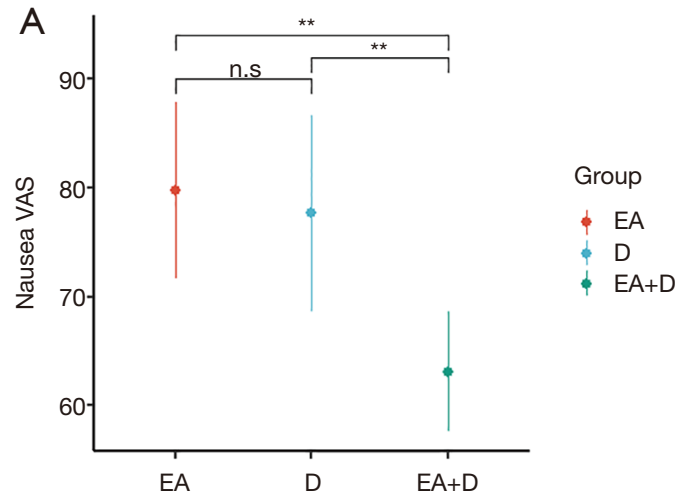

B

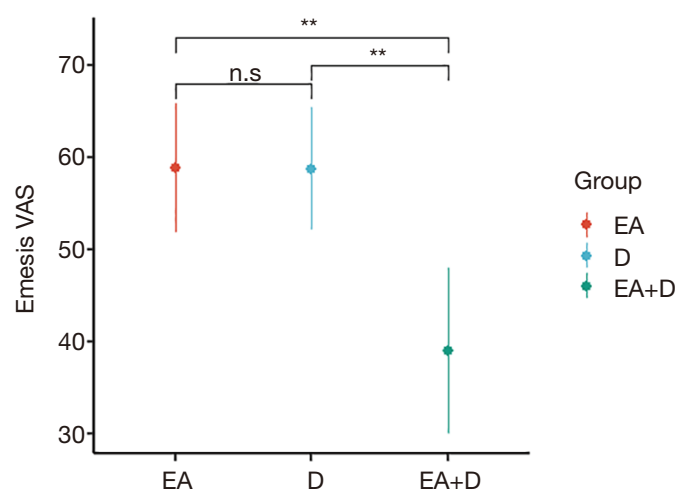

Figure 6 VAS scores of nausea and emesis at the end of gastroscopy among groups: (A) VAS score of nausea, PEA-D $=0.18$, $\mathrm{PEA}+\mathrm{D}-\mathrm{D}=4.1 \mathrm{e}-13, \mathrm{PEA}+\mathrm{D}-\mathrm{EA}=7.1 \mathrm{e}-14$; (B) VAS score of emesis, PEA-D=0.99, PEA+D-D=9.9e-15, PEA+D-EA=2.0e-14. The VAS scale was used to immediately evaluate the two indicators after gastroscopy, and all data results were expressed as mean \pm standard deviation $\left(\bar{x}_{ \pm} \mathrm{SD}\right)$. Comparison of the $\mathrm{EA}+\mathrm{D}$ group with the EA and $\mathrm{D}$ groups: ${ }^{* *} \mathrm{P}<0.01$; comparison of the $\mathrm{EA}$ and $\mathrm{D}$ groups: n.s. $\mathrm{P}>0.05$.

and a series of psychological and physiological reactions and damage through the endocrine pathways, including abnormal gastric motility, changes in gastric electrical activity, mucosal damage, and changes in gastric acid and gastrointestinal hormones, and accordingly causes a series of clinical symptoms (2). Extensive clinical literature and numerous reports consider that acupuncture, moxibustion, and EA can alleviate the side effects of gastroscopy (13-16). Furthermore, the results of meta-analyses of the literature reveal that acupuncture can alleviate the side effects of gastroscopy to a certain extent $(13,19)$.

EA combines traditional Chinese medicine and modern treatment. First, a needle is inserted into an acupoint to

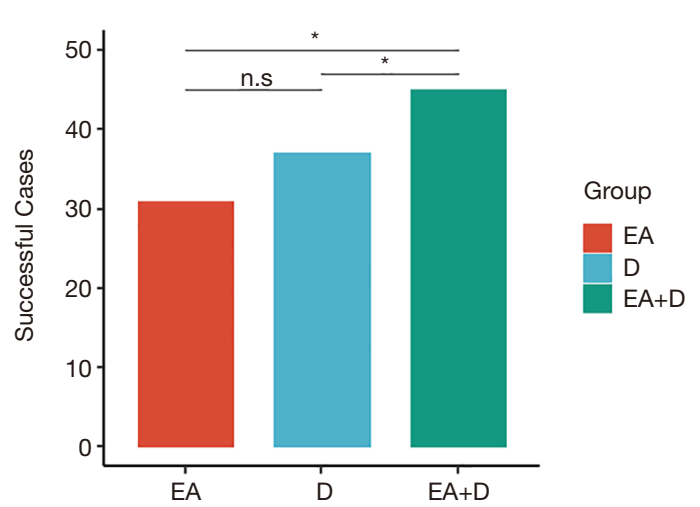

Figure 7 Comparison of the number of successful cases of one-stop intubation in gastroscopy in all groups. The data of "the number of successful cases of one-stop intubation" were expressed as n (patients). PD:EA=0.297000, PD: $\mathrm{EA}+\mathrm{D}=0.000348$, PEA:EA+D=0.012700. Comparison of the $E A+D$ group with the EA and D groups: ${ }^{*} \mathrm{P}<0.05$; comparison of the EA and $\mathrm{D}$ groups: n.s. $\mathrm{P}>0.05$.

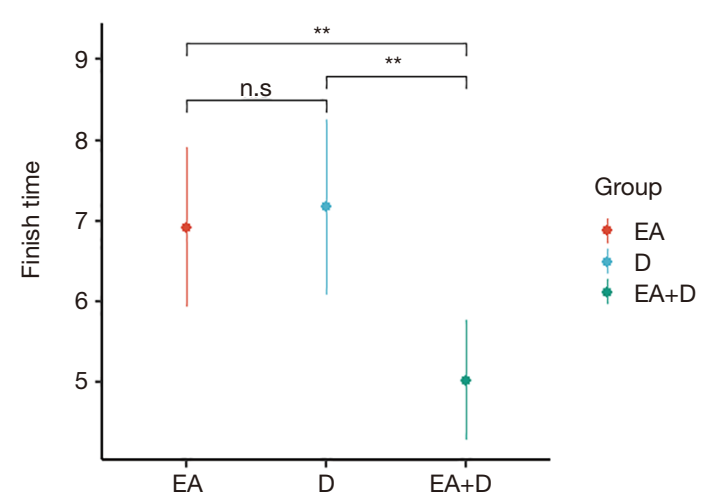

Figure 8 Comparison of duration of gastroscopy among the three groups. The duration of gastroscopy (min) was measured using a stopwatch. The results were rounded, and the data units were calculated in approximate minutes. PEA-D=0.12, PEA+D$\mathrm{D}=5.4 \mathrm{e}-14, \mathrm{PEA}+\mathrm{D}-\mathrm{EA}=8.3 \mathrm{e}-14$; All data results were expressed as mean \pm standard deviation $\left(\bar{x}_{ \pm} \mathrm{SD}\right)$. Comparison of the $\mathrm{EA}+\mathrm{D}$ group with the $\mathrm{EA}$ and $\mathrm{D}$ groups: ${ }^{* *} \mathrm{P}<0.01$; comparison of the $\mathrm{EA}$ and $\mathrm{D}$ groups: n.s. $\mathrm{P}>0.05$.

bring about the desired sensation. Then, the needle is connected with a microcurrent close to the bioelectricity of the human body. Thus, this method is a combination of needling and electric stimulation; it has the potential to prevent and treat diseases $(20,21)$. To date, the literature reports that EA can adjust human physiological functions (22), relieve pain (23), sedate (24), promote 
blood circulation (25), and adjust muscle tension (26). The applicability of EA is essentially the same as that of acupuncture needling; it therefore has a wide range of treatment applications. In clinic, it is often used in pain syndromes $(23,27)$; arthralgia syndrome (28); dysfunction of the stomach, intestine, and other organs (29,30); mania (31); muscle (26), ligament (23), and joint (28) damage; and for acupuncture anesthesia (32). However, there is no straightforward research data related to the use of EA for the prevention of side effects in gastroscopy. Hence, there is a need to systematically and scientifically investigate the application of EA in the prevention and treatment of side effects of gastroscopy, enteroscopy, duodenoscopy, and ERCP, and to explore the relevant mechanisms. Furthermore, it will be of great significance to scientifically understand the role and mechanism of EA in preventing gastroscopy and colonoscopy side effects.

Based on existing literature and clinical experience, and with the consent and support of the Ethics Committee of our hospital, the researchers in the present study designed a clinical trial to investigate the effectiveness of EA combined with dyclonine hydrochloride mucilage in preventing the side effects of gastroscopy. The results of the present study reveal that incidences of side effects (such as nausea, emesis, salivation, restlessness, and breath holding) were lower in patients in the treatment group (the EA+D group, who received the combined treatment) than in patients receiving only one of the two treatments (in the EA group and the D group) $(\mathrm{P}<0.05$ and $\mathrm{P}<0.01$, respectively). Furthermore, as the gastroscope passed through the pylorus and after the examination, the changes in $\mathrm{HR}$ and $\mathrm{SpO}_{2}$ were better in the $\mathrm{EA}+\mathrm{D}$ group than in the EA group and the $\mathrm{D}$ group $(\mathrm{P}<0.05$ and $\mathrm{P}<0.01$, respectively), and the VAS scores for nausea and emesis, the first-pass success rate, and examination duration were better for the $\mathrm{EA}+\mathrm{D}$ patients than for those in the EA group and the $\mathrm{D}$ group $(\mathrm{P}<0.05$ and $\mathrm{P}<0.01$, respectively). This series of results suggests that $\mathrm{EA}+\mathrm{D}$ can alleviate the above-mentioned nerve, heart, and gastrointestinal stress responses induced by gastroscopy. Furthermore, the results of the present study are basically in agreement with those in the literature, where it is reported $(13,19)$ that acupuncture at Zusanli before gastroscopy can relieve nausea and emesis during the procedure. Researchers who have reviewed and analyzed the application of therapies of traditional Chinese medicine (such as acupuncture, EA, moxibustion, and acupoint injection) in upper gastrointestinal endoscopy conclude that acupuncture (including EA, acupoint massage, and moxibustion) can alleviate anxiety and adverse gastrointestinal reactions by regulating nervous and gastrointestinal electrical activity. They also found that the treatments of traditional Chinese medicine supported reductions in the dosage of anesthetics given to patients and promoted a shorter awakening time from anesthesia (14-16).

Among the literature related to the mechanism of EA and acupuncture in preventing the side effects of gastroscopy and colonoscopy, one previous study reports that acupuncture relieved a series of uncomfortable symptoms or sign changes caused by gastroscopy, and mitigated changes in mechanisms, pathways, signals, and transmitters. Studies have also revealed that acupuncture and moxibustion can regulate vegetative nerve and gastrointestinal function, protect gastric mucosa, promote resistance to anxiety, and reduce the concentration of 5-HT in blood (33); it follows that acupuncture and moxibustion can reduce emesis as a side effect during gastroscopy. Similar mechanisms can be found for other side effects. Acupuncture and moxibustion can influence the biological factors of anatomy and physiology. The mechanism of EA in preventing the side effects of gastroscopy is correlated to this. However, the stimulation intensity of EA is greater than that of simple acupuncture, and further clinical and animal studies are needed to verify whether there are other mechanisms involved, or whether the regulatory effect of EA on the secretion of neurosubstances is stronger than that of acupuncture alone.

One study involving an animal experiment reveals that the mechanism of the regulatory effect of EA on the gastrointestinal tract may be correlated to the inhibition of stimulation of the limbic system. For example, when EA was given at Zusanli and other acupoints, electrophysiological activities and blood flow and velocity increased in the gastrointestinal tract tissues of the experimental animals (26,27). In another experimental study (on dogs) (28), changes in the upper gastrointestinal tract of dogs after EA treatment at the Zusanli were characterized by a decrease in total pressure, basal pressure, and contraction frequency of the pyloric sphincter. The expression of calcitonin and related genes in plasma and the gastric mucosa of dogs was also detected, and the results reveal that the concentrations of these were higher in the treatment group than in the control group, whereas the concentration of endothelin was significantly lower. The preliminary results of the present study reveal that EA can regulate ghrelin to a certain extent, thereby regulating the gastrointestinal-neurophysiological reflex function. 
The limitations of the present study should be mentioned. This was a small-scale study involving a small sample. There is no previous similar study to support our sample size calculation; this is therefore an exploratory study. The data obtained in this study will support the sample size calculations of future multi-center and largescale clinical trials. Based on clinical trials, the present study aimed to determine the pathway of action of acupuncture and the changes in the corresponding indicator transmitters. It is only through this approach that powerful evidencebased medical evidence can be obtained. Because our study was a small-sample, prospective cohort study, there is the potential for bias (e.g., selection bias, performance bias, and measurement bias). In the future, multi-center and largescale clinical trials are needed to eliminate such bias.

\section{Conclusions}

On the basis of the present study and the literature, the researchers consider that acupuncture can alleviate the side effects induced by endoscopy. The associated advantages are the simplicity, convenience, and economy of acupuncture, and the potential to reduce the side effects of drugs; furthermore, the treatment has strong operability in clinic and can therefore be easily accepted by patients.

\section{Acknowledgments}

Funding: This study was supported by National Natural Science Foundation of China [No.81673934, 81973608, 62041701], the Project of the 13th Five-Year Plan of China [No.2017ZX10305501], 2018 Natural Science Foundation of Beijing [No.7192083], China Postdoctoral Science Foundation [No.2019M650774], the Project of The 81th Group Army Hospital of the Chinese People's Liberation Army [No.KT201603, No.KT2015003], the New Technology and New Business of The 81th Group Army Hospital of the Chinese People's Liberation Army [No.JS2016026] and 2019 Natural Science Foundation of Capital Medical University [No.PYZ19170].

\section{Footnote}

Reporting Checklist: The authors have completed the STRICTA reporting checklist. Available at http://dx.doi. org/10.21037/apm-20-831

Data Sharing Statement: Available at http://dx.doi. org/10.21037/apm-20-831

Conflicts of Interest: All authors have completed the ICMJE uniform disclosure form (available at http://dx.doi. org/10.21037/apm-20-831). The authors have no conflicts of interest to declare.

Ethical Statement: The authors are accountable for all aspects of the work in ensuring that questions related to the accuracy or integrity of any part of the work are appropriately investigated and resolved. The study was conducted in accordance with the Declaration of Helsinki (as revised in 2013). The study was approved by the ethics committee of the 81th Group Army Hospital of the Chinese People's Liberation Army (No.: 2016JL03-013-01) and informed consent was taken from all the patients.

Open Access Statement: This is an Open Access article distributed in accordance with the Creative Commons Attribution-NonCommercial-NoDerivs 4.0 International License (CC BY-NC-ND 4.0), which permits the noncommercial replication and distribution of the article with the strict proviso that no changes or edits are made and the original work is properly cited (including links to both the formal publication through the relevant DOI and the license). See: https://creativecommons.org/licenses/by-nc-nd/4.0/.

\section{References}

1. Chandrasekhara V, Elmunzer BJ, Khashab M, et al. Clinical Gastrointestinal Endoscopy. 3rd ed. Berkeley: Elsevier, 2018:2-11.

2. Keeffe EB. Towards safer endoscopy. In: Cotton PB, Tytgat GNJ, Williams CB, et al. editors. Annual of gastrointestinal endoscopy. London: Rapid Science Publisher, 1997:1-14.

3. Borison HL, Borison R, McCarthy LE. Role of the area postrema in vomiting and related functions. Fed Proc 1984;43:2955-8.

4. Ozel AM, Oncü K, Yazgan Y, et al. Comparison of the effects of intravenous midazolam alone and in combination with meperidine on hemodynamic and respiratory responses and on patient compliance during upper gastrointestinal endoscopy: a randomized, double-blind trial. Turk J Gastroenterol 2008;19:8-13.

5. Hayward SR, Sugawa C, Wilson RF. Changes in oxygenation and pulse rate during endoscopy. Am Surg 1989;5 5:198-202. 
6. Saijyo T, Nomura M, Nakaya Y, et al. Assessment of autonomic nervous activity during gastrointestinal endoscopy: analysis of blood pressure variability by tonometry. J Gastroenterol Hepatol 1998;13:816-20.

7. Campo R, Brullet E, Montserrat A, et al. Efficacy of low and standard midazolam doses for gastroscopy. A randomized, double-blind study. Eur J Gastroenterol Hepatol 2000;12:187-90.

8. Froehlich F, Schwizer W, Thorens J, et al. Conscious sedation for gastroscopy: patient tolerance and cardiorespiratory parameters. Gastroenterology 1995;108:697-704.

9. Trevisani L, Sartori S, Gaudenzi P, et al. Upper gastrointestinal endoscopy: are preparatory interventions or conscious sedation effective? A randomized trial. World J Gastroenterol 2004;10:3313-7.

10. Mulcahy HE, Greaves RR, Ballinger A, et al. A doubleblind randomized trial of low-dose versus high-dose topical anaesthesia in unsedated upper gastrointestinal endoscopy. Aliment Pharmacol Ther 1996;10:975-9.

11. Iber FL, Sutberry M, Gupta R, et al. Evaluation of complications during and after conscious sedation for endoscopy using pulse oximetry. Gastrointest Endosc 1993;39:620-5.

12. Mokhashi MS, Hawes RH. Struggling toward easier endoscopy. Gastrointest Endosc 1998;48:432-40.

13. Lazzaroni M, Bianchi Porro G. Preparation, premedication, and surveillance. Endoscopy 2005;37:101-9.

14. Cahn AM, Carayon P, Hill C, et al. Acupuncture in gastroscopy. Lancet 1978;1:182-3.

15. Tarçin O, Gürbüz AK, Poçan S, et al. Acustimulation of the neiguan point during gastroscopy: its effects on nausea and retching. Turk J Gastroenterol 2004;15:258-62.

16. Lee H, Ernst E. Acupuncture for GI endoscopy: a systematic review. Gastrointest Endosc 2004;60:784-9.

17. Committee for the Examination and Approval of Terms of Traditional Chinese Medicine. Terms of Traditional Chinese Medicine. Beijing: Science Press, 2005.

18. Yuan JH, Li XM. Clinical study on prevention and treatment of stress response by gastroscopy in middleaged and elderly patients by psychological intervention combined with percutaneous acupoint electrical stimulation. Hebei Med 2012;34:3017-8.

19. Wang W, Zhang T, Peng W, et al. Acupuncture for discomfort in patients during gastroscopy: a systematic review protocol. BMJ Open 2014;4:e005735.
20. Ye Q, Liu C, Shi J, et al. Effect of electro-acupuncture on regulating the swallowing by activating the interneuron in ventrolateral medulla (VLM). Brain Res Bull 2019;144:132-9.

21. Zhang R, Andersen AH, Hardy PA, et al. Objectively measuring effects of electro-acupuncture in parkinsonian rhesus monkeys. Brain Res 2018;1678:12-19.

22. Ulloa L, Quiroz-Gonzalez S, Torres-Rosas R. Nerve Stimulation: Immunomodulation and Control of Inflammation. Trends Mol Med 2017;23:1103-20.

23. Grech D, Li Z, Morcillo P, et al. Intraoperative Lowfrequency Electroacupuncture under General Anesthesia Improves Postoperative Recovery in a Randomized Trial. J Acupunct Meridian Stud 2016;9:234-41.

24. Liu HF, Zhao Z, Zhang J, et al. Acupuncture reduces nicotine-induced norepinephrine release in the hypothalamus via the solitary NMDA receptor/NOS pathway. Neurosci Lett 2019;705:33-8.

25. Kamali F, Mirkhani H, Nematollahi A, et al. The Effect of Transcutaneous Electrical Nerve Stimulation of Sympathetic Ganglions and Acupuncture Points on Distal Blood Flow. J Acupunct Meridian Stud 2017;10:120-124.

26. Zhang R, Lao L, Ren K, et al. Mechanisms of acupuncture-electroacupuncture on persistent pain. Anesthesiology 2014;120:482-503.

27. Shin S, Yang SP, Yu A, et al. Effectiveness and safety of electroacupuncture for poststroke patients with shoulder pain: study protocol for a double-center, randomized, patient- and assessor-blinded, sham-controlled, parallel, clinical trial. BMC Complement Altern Med 2019;19:58.

28. Lewis J, Sim J, Barlas P. Acupuncture and electroacupuncture for people diagnosed with subacromial pain syndrome: A multicentre randomized trial. Eur J Pain 2017;21:1007-19.

29. Wu HG, Jiang B, Zhou EH, et al. Regulatory mechanism of electroacupuncture in irritable bowel syndrome: preventing MC activation and decreasing SP VIP secretion. Dig Dis Sci 2008;53:1644-51.

30. Zhou J, Liu Y, Zhou K, et al. Electroacupuncture for Women with Chronic Severe Functional Constipation: Subgroup Analysis of a Randomized Controlled Trial. Biomed Res Int 2019;2019:7491281.

31. Wang Z, Wang X, Liu J, et al. Acupuncture treatment modulates the corticostriatal reward circuitry in major depressive disorder. J Psychiatr Res 2017;84:18-26.

32. Wu L, Liu X, Yin Y, et al. Effectiveness of acupuncture 
versus spinal-epidural anesthesia on labor pain: a randomized controlled trial. J Tradit Chin Med 2017;37:629-35.

Cite this article as: Chen JM, Li DD, Chen YS, Lian B, Wang XP, Guo YH, Xu XL, Huang P, Chen TF, Liu Y, Liu QQ. The effectiveness of electro-acupuncture combined with dyclonine hydrochloride in relieving the side effects of gastroscopy: a controlled trial. Ann Palliat Med 2021;10(3):29582970. doi: 10.21037/apm-20-831
33. Xie YD, Yang Q, Wang JJ, et al. Study on the regulating effect of electro-acupuncture at Zusanli point on gastric electricity in rats. J Shanxi Med Univ 2011;42:202-4. 